\title{
GIS as Project Manager in Geophysical Applications Software
}

\author{
Khalid Amin Khan ${ }^{1}$, Gulraiz Akhter ${ }^{2}$, Zulfiqar Ahmad ${ }^{2}$ \\ ${ }^{1}$ K-tron Research Inc., Rawalpindi, Pakistan \\ ${ }^{2}$ Deptt. of Earth Sciences, Quaid-i-Azam University, Islamabad, Pakistan \\ E-mail:aminkhan@k-tron.net \\ Received January 12, 2011; revised January 24, 2011; accepted February, 9, 2011
}

\begin{abstract}
The changing trends in information technology have greatly influenced the role of GIS in spatial data management, analysis, processing and presentation. It has evolved from the conventional cartography and image processing to advanced 3D visualization and dynamic graphics tools. Due to this evolving nature of GIS, it has found wide applications in a number of diverse fields. Geophysical exploration projects involve data acquisition at hundreds of spatial locations resulting in large number of datasets. It takes a great deal of time to manage all these datasets during data processing and interpretation. This paper presents the use of GIS as an effective project management tool, providing an interactive data access interface in compute intensive geophysical processing applications. A reusable GIS software component is presented which can be used by geophysical applications to manage their datasets. A practical example is included to demonstrate the implementation of this GIS component as an embedded Project Manager in a seismic refraction software.
\end{abstract}

Keywords: GIS, Project Manager, Interactive Interface, Database, Geophysics

\section{Introduction}

GIS is a graphical tool for providing access to geo-referenced data. It has been broadly classified into two types: Raster based GIS and Vector based GIS. The former involves image processing techniques for handling satellite imagery and remote sensing data, while the later uses relational database management systems (RDBMS) for storage of data along with geographic co-ordinates and attributes which are translated into vector graphics for GIS presentation. Current systems are based on merger of the two types, where satellite imagery is overlaid by multiple layers of vector graphics data.

GIS has been successfully used in a number of areas. Some of the conventional uses include; geological mapping [1,2] environment [3], waste management [4], meteorology [5], defence, urban growth [6], traffic flow analysis [7], health, planning, ecology and animal habitat [8], agriculture, forestry [9], soil erosion [10], earthquake [11], hazard management [12], tunnel analysis [13], digital elevation models [14,15], soil analysis [16], minerals resources [17], geothermal resource exploration [18], glaciology [19], hydrology and ground water ex- ploitation [20-22].

In addition to the above applications there is an expanding scope of GIS in new areas. This paper presents the development of a GIS Project Manager software component that can be used in geophysical applications to manage project datasets. The concept has been demonstrated through a practical example by implementing GIS as an embedded Project Manager in a seismic refraction software.

\section{GIS Coupled with Processing Applications}

With the advent of advanced graphics, large memory systems and storage media technologies the role of GIS has further widened. Attempts have been made to incorporate the emerging technologies with GIS and extend their role and functionality in different areas.

GIS being a general-purpose tool is used in a number of fields each having specific processing requirements, which are catered through separate processing software. It is beyond the scope of GIS to incorporate processing tools for all fields in which GIS is used. The solution to this requirement is achieved by linking GIS with spe- 
cialized processing software through an inter-process communication mechanism called Remote Procedure Call (RPC) [23]. This link allows data collected at spatial locations and stored in GIS to be dynamically passed to the processing application. The current study mainly involves advancements in this direction; therefore it is discussed in more detail.

It has been realized that a complete GIS solution should have a GIS graphic front-end, RDBMS and related processing applications (PA) [24]. PA can be implemented with GIS through the following two approaches [25]:

- Loosely-coupled Approach: PA and the GIS are executed independent from each other and are linked through transfer of data. This link is established through data formats between GIS and PA [26].

- Tightly-coupled Approach: Either PA must be built within the GIS or the GIS functionality must be built within the PA.

The selection of either of the above two approaches depends on the complexity of geophysical PAs, volume of data involved and required memory resources, and the degree of interaction desired between the GIS and PA.

\section{GIS in Geophysical Applications}

Geophysical investigations usually involve large volumes of diverse data and compute intensive processing algorithms. With the advent of 3D geophysical surveys the data volume has further increased, resulting in large number of datasets. Moreover, the processing of these datasets also involves several supporting data types. In case of seismic data processing, apart from seismic data, there are geometry, navigation, statics, velocity and job control datasets. Geophysical data is acquired at several random or equidistant locations within the survey area. Thus in a project there are several data acquisition points each having more than one datasets. For a single project there are hundreds of files and all of them must be loaded into the system for processing. Furthermore various processing stages may also generate several output datasets. Thus it becomes very difficult for the geophysical analyst to maintain and manage all the datasets used in a project. There is a need for a GIS based data management technology, which keeps track of all the input and output datasets along with their processing status and provides and interactive interface to access these datasets.

GIS is commonly used in seismic interpretation software to view the base map and get interactive access to seismic sections, but such tools are not available for other geophysical methods. Thus a general purpose GIS Project Manger component is required which can be used in seismic refraction, gravity, magnetic, resistivity and other exploration related software. To implement a GIS based Project Manager, the tightly-coupled approach has been adopted. Within this approach there are two options for incorporating GIS functionality in PAs.

- Extended Tool Boxes: PAs are invoked from within the GIS as it's extended tools.

- Embedded GIS: Selected GIS functions are built in the PA.

Geophysical problems are too complex and compute intensive and therefore cannot be implemented as toolboxes within the GIS, thus the embedded GIS concept is used to develop a component for geophysical applications.

\section{GIS Project Manager Component}

The required GIS Project Manager functionality has been implemented in the form of an AciveX [27] software component that can be used by geophysical processing applications. It is basically an object oriented reusable component which provides a set of properties, methods and events to customize, control and process data access. It includes a prebuilt GIS and Project Explorer which can be customized, by setting component properties according to the requirements of a geophysical application. It generates and maintains the project database in Microsoft Access format. The component methods and an event are listed in Table 1 along with their functionality. They are used by the geophysical application to manage and access data. This component can be implemented in any geophysical software developed in a programming language which supports Component Object Model (COM) [28].

The GIS Project Manager has three essential sub-components: Project Database, GIS as the core technology, and Project Explorer. The functionality of these components is briefly discussed below, followed by detailed working of the component.

\subsection{Project Database}

The project database is basically a relational database management system (RDBMS). It stores all user specified parameters, data processing job sequence, filenames of all input and output datasets involved in the project as well as navigation data files for lines coordinates. In addition it also maintains the processing status of each dataset. The project database tables and their fields are shown in Figure 1. The AppID table is used for applica- 
Table 1. Methods and an Event Procedure of GIS Project Manager component along with their functionality.

\begin{tabular}{|c|c|}
\hline Methods & Function \\
\hline SetAppInfo (AppName, AppVer) & \multirow{2}{*}{ Set/Get Application identification information } \\
\hline GetAppInfo (AppName, AppVer) & \\
\hline SetGenInfo (InfoTag, InfoValue) & \multirow{2}{*}{ Set/Get General Information } \\
\hline GetGenInfo (InfoTag, InfoValue) & \\
\hline AddNavData (LineType, NavFile) & \multirow{2}{*}{ Add/Delete Navigation Data Files } \\
\hline DelNavData (NavFile) & \\
\hline AddGeoData (LineName, Picket, GeoType, GeoFile) & \multirow{2}{*}{ Add/Delete Geoscientific Data Files at specified Line and Picket } \\
\hline DelGeoData (LineName, Picket) & \\
\hline SetProcessStatus (LineName, Picket, ProStatus) & \multirow{2}{*}{ Set/Get Processing Status of Geoscientific Data at a Line Picket } \\
\hline GetProcessStatus (LineName, Picket, ProStatus) & \\
\hline SetViewStatus (LineName, Picket, ViewStatus) & Set View Status to highlight Picket on the GIS and Project Explorer \\
\hline SetSymbol(GeoType, Symbol) & Set Picket Symbols for various Geoscientific Data Types \\
\hline AddDataset (DatType, DatFile) & \multirow{2}{*}{ Add/Delete any optional Data Files } \\
\hline DelDataset (DatFile) & \\
\hline SetPara (ParaTag, ParaValue) & \multirow{2}{*}{ Set/Get any optional Processing Parameters } \\
\hline GetPara (ParaTag, ParaValue) & \\
\hline Event Procedure & Function \\
\hline GISPM_CallGeoData (LineName, Picket, GeoType,_ & Event Triggered from GIS or Project Explorer to access Geophysical \\
\hline GeoFile, ProStatus, Button) & Data and Call Application’s Data View Port \\
\hline
\end{tabular}

\begin{tabular}{|c|}
\hline AppID \\
\hline AppInfo \\
\hline $\begin{array}{l}\text { AppName } \\
\text { AppVersion }\end{array}$ \\
\hline
\end{tabular}

GenInfo

\begin{tabular}{|l|l|}
\hline InfoTag & InfoValue \\
\hline$<$ Project $>$ & \\
$<$ Company $>$ & \\
$<$ Crew $>$ & \\
$<$ Client $>$ & \\
$<$ ProcessedBy $>$ & \\
$<$ StartDate $>$ & \\
$<$ EndDate $>$ & \\
\hline
\end{tabular}

NavData

\begin{tabular}{|l|l|}
\hline LineType & NavFile \\
\hline & \\
\hline
\end{tabular}

GeoData

\begin{tabular}{|l|l|l|l|l|}
\hline LineName & Picket & GeoType & GeoFile & ProStatus \\
\hline & & & & \\
& & & & \\
\hline
\end{tabular}

Datasets
\begin{tabular}{|l|l|}
\hline DatType & DatFile \\
\hline & \\
& \\
\hline
\end{tabular}

Parameters

\begin{tabular}{|l|l|}
\hline ParaTag & ParaValue \\
\hline & \\
\hline
\end{tabular}

Figure 1. Project database tables and their fields. These tables are created and updated with information, parameters and filenames using the component methods. tion identification and the GenInfo table is used to store tag based general project information. Any number of unique tags names can be introduced to store and retrieve information. The two main tables are; NavData for storing navigation data filenames and GeoData for storing geophysical data filenames along with their line names, picket numbers and processing status. Finally two optional tables, Datasets and Parameters, are available for storing any additional filenames and processing parameters respectively. For a new project, this database is created and updated by using the component methods for assigning processing parameters and storing filenames of all the project datasets, along with their respective data types.

\subsection{GIS Map}

The GIS is a front-end component of Project Manager. It displays a project map, showing the geographic location of all datasets along with their processing status. The datasets are presented on the GIS in the form of different graphic objects like box, rectangle, polygon, circle or other symbols each representing a different geophysical data type and their color indicates the current view status. Similarly output datasets created after processing are also 
indicated on the map. The GIS also provides an interactive interface through which the graphics objects on the GIS are hyperlinked to their associated datasets. Simply clicking a graphics object provides access to its associated datasets which are loaded, processed and displayed by their corresponding geophysical application. In this way the GIS not only displays the spatial distribution and processing status of data points in the project area, but also provides a direct access to their associated datasets.

\subsection{Project Explorer}

Another front-end component of Project Manager is the
Project Explorer, which displays a well organized data tree. It lists all project datasets under their respective data types. All acquisition lines and their pickets at which geophysical data is loaded are also given in the tree. Similar to the GIS, it also provides a direct access to each dataset listed in the tree.

\subsection{Working Procedure}

The functional diagram of GIS Project Manager component is given in Figure 2. Project information, processing parameters and datasets filenames are loaded into the project database using the component methods. Similarly

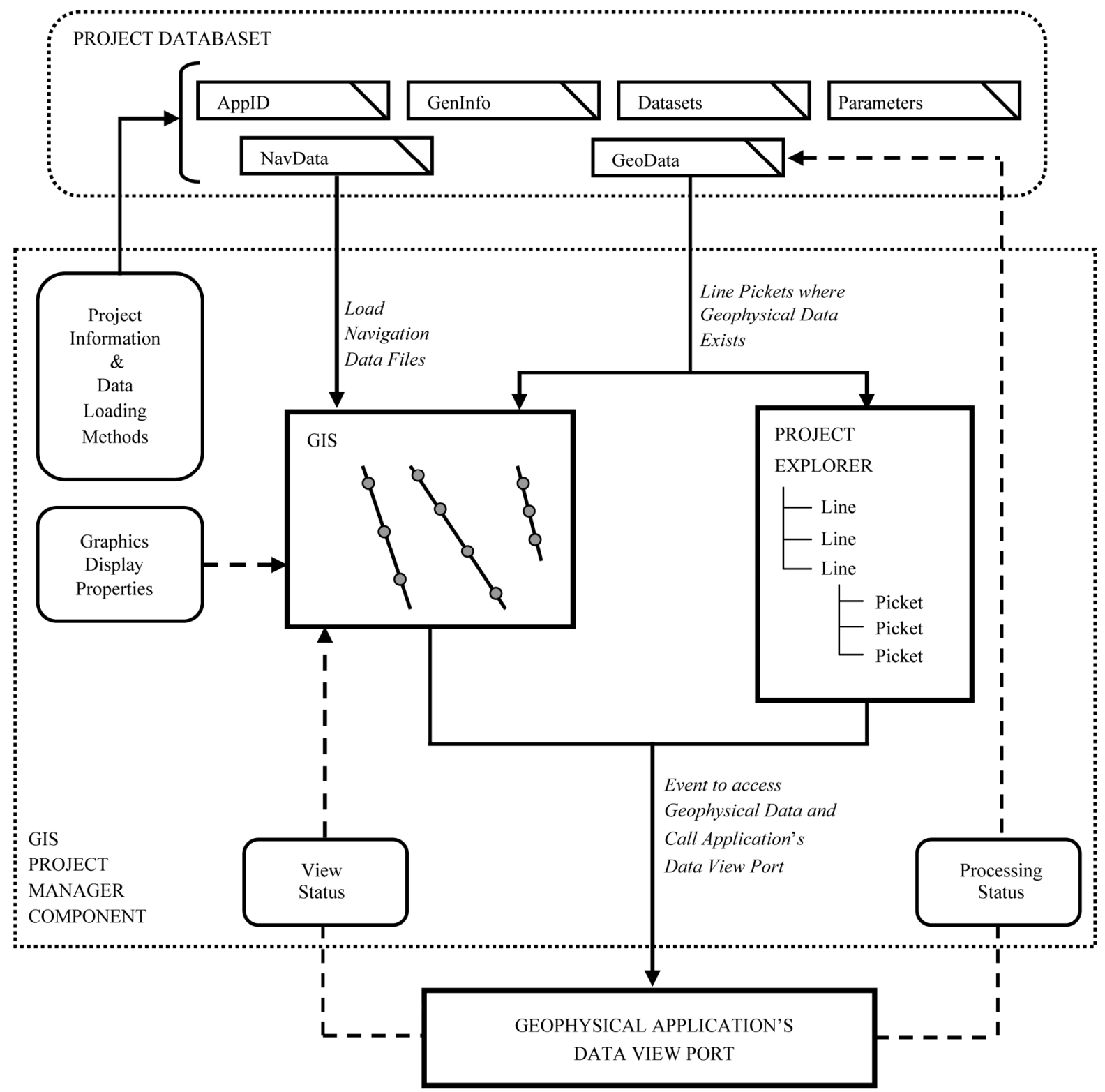

Figure 2. The complete functional diagram of the GIS Project Manager component along with Project Database and Application's Data View Port. 
the GIS display is customized by setting various properties. When a project database is opened, lines and pickets information is retrieved from the related database tables and displayed on the GIS and Project Explorer. Clicking any picket on the GIS or Project Explorer retrieves the associated geophysical datasets filenames from the table and activates an event procedure returning the line name, picket number, dataset filenames and their status as arguments. This event procedure in turn is used to read the geophysical datasets, by calling functions from related I/O library, and display them into the geophysical application's view port. Thus the GIS and Project Explorer provide a highly interactive access to the project datasets. Once the opened data is processed, its status is stored into the database, which in turn updates the GIS and Project Explorer. Using this mechanism multiple datasets, at various pickets, can be opened, displayed and processed. This component also keeps track of the datasets being displayed or viewed and reflects this on the GIS and Project Explorer. Thus it provides a three-way link between the front-end interfaces; GIS, Project Explorer and Geophysical Application's Data View Port. This link has a one-to-one relationship between graphics objects displayed on the GIS, datasets pickets listed in the Project Explorer tree and their corresponding Data View port. User actions or processing tasks causing changes in any one interface directly result in changes in the other two interfaces. Thus the GIS Project Manager provides a
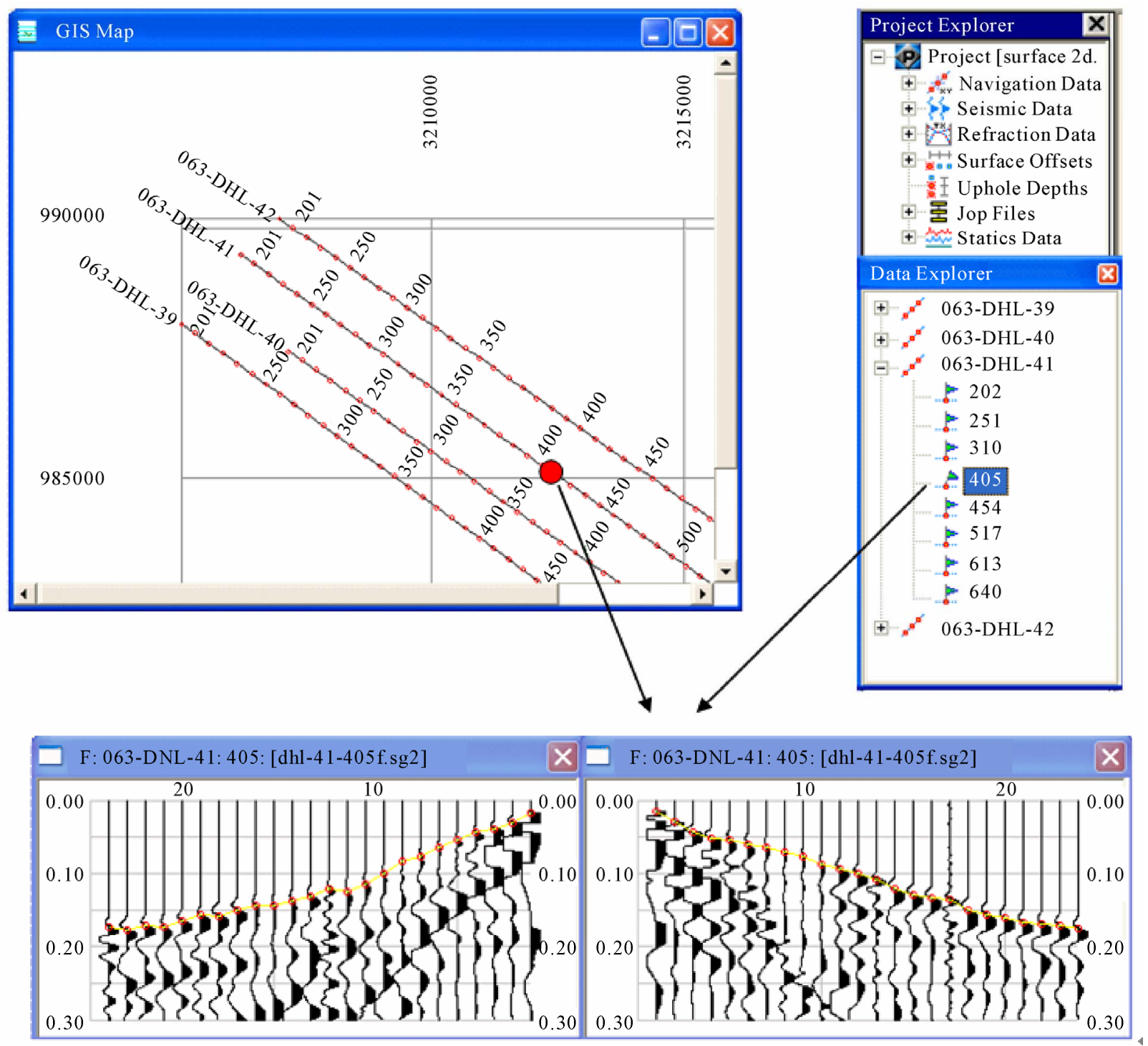

Figure 3. (Top-Left) Base Map of seismic lines displayed by GIS. (Top-Right) Project Explorer showing a tree of various Data Types and Data Objects (Seismic Lines) and their Refraction Pickets. Clicking a picket on the GIS Map or the Project Explorer loads and processes the associated seismic data (Bottom). 
highly interactive and efficient mechanism to access, view and process geophysical datasets in a large project.

\section{Implementation Example}

The GIS Project Manager component has been successfully implemented in a seismic refraction data processing software, which is a three stage application for picking arrival times, computing refractor model and finally calculating statics. A new project is created and all project datasets and processing parameters are defined into its database. Using the GIS or Project Explorer any dataset can be interactively opened and processed according to the predefined job sequence (Figure 3 ). In this way the Project Manager acts as an efficient data management tool in handling large seismic exploration projects. Another advantage of the Project Manager is that once all datasets have been defined in its database, there is no need to load them over and over again. Whenever the project data needs to be viewed or reprocessed, simply loading the project database provides full access to all defined datasets.

\section{Conclusions}

A GIS Project Manager component is presented which can be used by geophysical software applications dealing with seismic refraction, gravity, magnetic or electrical resistivity data. It provides a user-friendly interface for managing large geophysical exploration projects with several datasets and processing tasks. Datasets are accessed directly from the GIS, without using the conventional menus, thus saving a lot of user time. In addition the geographic location, type and status of all datasets involved in the project are directly shown on the GIS, which provides a complete picture of the project in terms of spatial distribution and processing status. The GIS based Project Manager is an effective and efficient tool for interactive and integrated data management of large geophysical projects.

\section{References}

[1] S. Dey and P. Ghosh, 2008, "GRDM-A Digital FieldMapping Tool for Management and Analysis of Field Geological Data,” Computers \& Geosciences, Vol. 34, No. 5, 2008, pp. 464-478. doi:10.1016/j.cageo.2007.05.014

[2] S. P. Colman-Sadd, J. S. Ash and L. W. Nolan, "GeoLegend: A Database System for Managing Geological Map Units in a Geographic Information System," Computers and Geosciences, Vol. 23, No. 7, 1997, pp. 715-724. doi:10.1016/S0098-3004(97)00069-1

[3] R. J. Viger, “The GIS Weasel: An Interface for the De- velopment of Geographic Information Used in Environmental Simulation Modeling," Computers \& Geosciences, Vol. 34, No. 8, 2008, pp. 891-901. doi:10.1016/j.cageo.2007.07.004

[4] R. S. Muttiah, B. A. Engel and D. D. Jones, "Waste Disposal Site Selection Using GIS-Based Simulated Annealing,” Computers and Geosciences, Vol. 22, No. 9, 1996, pp. 1014-1017.

[5] J. Massons, D. Domingo and J. Grau, "Automatic Classification of VIS-IR METEOSAT Images,” Computers and Geosciences, Vol. 22, No. 10, 1996, pp. 1137-1146. doi:10.1016/S0098-3004(96)00058-1

[6] W. Acevedo and P. Masuoka, "Time-Series Animation Techniques for Visualizing Urban Growth,” Computers and Geosciences, Vol. 23, No. 4, 1997, pp. 423-435. doi:10.1016/S0098-3004(97)00006-X

[7] B. Jiang and C. Liu, "Street-Based Topological Representations and Analyses for Predicting Traffic Flow in GIS,” International Journal of Geographical Information Systems, Vol. 23, No. 9, 2009, pp. 1119-1137.

[8] O. McNoleg, "The Integration of GIS, Remote Sensing, Expert Systems and Adaptive Co-Kriging for Environmental Habitat Modelling of Highland Haggis Using Object-Oriented, Fuzzy Logic and Neural-Network Techniques,” Computers and Geosciences, Vol. 22, No. 5, 1996, pp. 585- 588. doi:10.1016/0098-3004(95)00131-X

[9] M. A. Wulder, “The Prediction of Leaf Area Index from Forest Polygons Decomposed through the Integration of Remote Sensing, GIS, UNIX, and C," Computers and Geosciences, Vol. 24, No. 2, 1998, pp. 151-157. doi:10.1016/S0098-3004(97)00132-5

[10] D. K. Molnar and P. Y. Julien, "Estimation of Upload Erosion Using GIS,” Computers and Geosciences, Vol. 24, No. 2, 1998, pp. 183-192. doi:10.1016/S0098-3004(97)00100-3

[11] E. K. Louvari and A. A. Kiratzi, "RAKE: A Windows Program to Plot Earthquake Focal Mechanisms and the Orientation of Principal Stresses," Computers and Geosciences, Vol. 23, No. 8, 1997, pp. 851-857. doi:10.1016/S0098-3004(97)00070-8

[12] C. Audisio, G. Nigrelli and G. Lollino, "A GIS Tool for Historical Instability Processes Data Entry: An Approach to Hazard Management in two Italian Alpine River Basins,” Computers and Geosciences, Vol. 35, No. 8, 2009, pp. 1735-1747. doi:10.1016/j.cageo.2009.01.012

[13] Y. Choi, S. Y. Yoon and H. D. Park, “Tunnelling Analyst: A 3D GIS Extension for Rock Mass Classification and Fault Zone Analysis in Tunnelling,” Computers and Geosciences, Vol. 35, No. 6, 2009, pp. 1322-1333. doi:10.1016/j.cageo.2008.05.002

[14] A. K. Skidmore, "A Comparison of Techniques for Calculating Gradient and Aspect from a Gridded Digital Elevation Model," International Journal of Geographical Information Systems, Vol. 3, No. 4, 1989, pp. 323-344.

[15] S. Kienzle, "The effect of DEM Raster Resolution on First Order, Second Order, and Compound Terrain Derivatives,” Transactions in GIS, Vol. 8, No. 1, 2004, pp. 
83-111. doi:10.1111/j.1467-9671.2004.00169.x

[16] T. S. Farewell and D. M. Farewell, "Knowledge-based Soil Attribute Mapping in GIS: Corrections and Extensions to the Expector Method," Transactions in GIS, Vol. 14, No. 2, 2010, pp. 183-192. doi:10.1111/j.1467-9671.2010.01190.x

[17] W. Zhou, G. Chen, H. Li, H. Luo and S. L. Huang, “GIS Application in Mineral Resource Analysis - A Case Study of Offshore Marine Placer Gold at Nome, Alaska," Computers \& Geosciences, Vol. 33, No. 6, 2007, pp. 773-788. doi:10.1016/j.cageo.2006.11.001

[18] Y. Noorollahi, R. Itoi, H. Fujii and T. Tanaka, “GIS Model for Geothermal Resource Exploration in Akita and Iwate Prefectures, Northern Japan,” Computers \& Geosciences, Vol. 33, No. 8, 2007, pp. 1008-1021. doi:10.1016/j.cageo.2006.11.006

[19] C. Knoll, H. Kerschner, A. Heller and P. Rastner, “A GIS-Based Reconstruction of Little Ice Age Glacier Maximum Extents for South Tyrol, Italy,” Transactions in GIS, Vol. 13, No. 5-6, 2009, pp. 449-463. doi:10.1111/j.1467-9671.2009.01173.x

[20] Y. Abdella and K. Alfredsen, "A GIS Toolset for Automated Processing and Analysis of Radar Precipitation,” Computers and Geosciences, Vol. 36, No. 4, 2010, pp. 422-429. doi:10.1016/j.cageo.2009.08.008

[21] W. H. Merkel, R. M. Kaushika and E. Gorman, "NRCS GeoHydro-A GIS Interface for Hydrologic Modeling," Computers and Geosciences, Vol. 34, No. 8, 2008, pp. 918-930. doi:10.1016/j.cageo.2007.05.020

[22] I. Portoghese, V. Uricchio and M. Vurro, “A GIS Tool for hydrogeological water balance evaluation on a regional scale in semi-arid environments," Computers \&
Geosciences, Vol. 31, No. 1, 2005, pp. 15-27. doi:10.1016/j.cageo.2004.09.001

[23] D. Cook, J. Symanzik, J. J. Majure and N. Cressie, “Dynamic Graphic in GIS: More Examples Using Linked Software," Computers and Geosciences, Vol. 23, No. 4, 1997, pp. 371-385. doi:10.1016/S0098-3004(97)00015-0

[24] K. A. Khan, "Integrated Geo Systems - A Computational Environment for Integrated Management, Analysis and Presentation of Petroleum Industry Data," In: T. C. Coburn and J. M. Yarus, Eds., Geographic Information Systems in Petroleum Exploration and Development, AAPG Book on Computers in Geology, No. 4, Chapter 16, 2000, pp. 215-226.

http://search.datapages.com/data/open/offer.do?target=\% 2Fspecpubs\%2Fca04\%2Fchap16\%2Fca04ch16.htm

[25] H. A. Karimi and B. J. Houston, "Evaluating Strategies for Integrating Environmental Models with GIS: Current Trends and Future Needs," Computers; Environment and Urban Systems, Vol. 20, No. 6, 1996, pp. 413-425. doi:10.1016/S0198-9715(97)00006-9

[26] C. G. Wesseling, D. J. Karssenberg, B. A. Burgh and W. P. A. Van Dearness, "Integrating Dynamic Environmental Models in GIS: The Development of a Dynamic Modelling Language,” Transactions in GIS, Vol. 1, No. 1, 1996, pp. 40-48. doi:10.1111/j.1467-9671.1996.tb00032.x

[27] S. D. Palmer, "Vbscript 2 \& Activex Programming: Master the Art of Creating Interactive Web Pages," 11 Edition, Coriolis Group Books, 1997, p. 8.

[28] D. Rogerson, “Inside COM,” Microsoft Press, Redmons, WA, 1996, pp. 159-210. 